\title{
DESASFALTAÇÃO E DESMETALIZAÇÃO DOS RESÍDUOS DE DESTILAÇÃO DO PETRÓLEO
}

\author{
I. F. COELHO ${ }^{1}$ e G. L. V. COELHO ${ }^{1}$ \\ ${ }^{1}$ Universidade Federal Rural do Rio de Janeiro, Departamento de Engenharia Química \\ E-mail para contato: coelho@ufrrj.br
}

\begin{abstract}
RESUMO - a necessidade de utilizar frações pesadas de petróleo de maneira eficiente na produção de frações mais nobres motiva a busca de meios para separar os asfaltenos, uma vez que esses contribuem para a formação do coque e para o envenenamento e desativação de catalisadores, além de provocar entupimentos e obstruções causados por sua deposição. Quando um apropriado solvente altera a condição de solvatação da estrutura coloidal rompendo o equilíbrio, ocorre uma floculação dando lugar a uma separação sólido/líquido e a consequente precipitação do asfalteno que carrega um alto teor de metais (Ni e Va). O propósito deste processo é também aplicar a desmetalização em resíduos de óleos, mostrando com mais clareza que as porfirinas metálicas presentes nos resíduos de óleos estão fortemente associadas com as moléculas asfaltênicas para formar aglomerados. Para enfraquecer esta associação faz-se necessário a introdução de uma substância solubilizante que rompa o equilíbrio do sistema e assim retire as resinas que protegem os asfaltenos associados com as porfirinas metálicas e permitia a aproximação dos asfaltenos até a sua precipitação. Pretendeu-se com este trabalho analisar a influência do agente precipitante nheptano e de solventes naftênicos e aromáticos no processo de desasfaltação do óleo cru pesado da Bacia de Campos, do campo de Marlim e também caracterizar por cromatografia o óleo devidamente desasfaltado.
\end{abstract}

\section{INTRODUÇÃO}

\subsection{O petróleo}

Os fornecimentos de óleos crus pesados têm aumentando a demanda por processos que sejam capazes de recuperar frações mais leves a partir de resíduos. Os asfaltenos estão entre os constituintes de óleos crus pesados que têm atraído interesse em nível mundial, pois apresentam grandes problemas técnicos, o que representa um desafio para a indústria do petróleo, sendo responsável por grandes perdas em etapas da produção de petróleo. Devido a isso, as operações de refino requerem consideráveis investimentos em unidades adicionais.

O petróleo pode ser definido, quanto à sua composição química, como uma mistura complexa de ocorrência natural, constituído predominantemente de hidrocarbonetos (podendo chegar a mais de $90 \%$ de sua composição) e não-hidrocarbonetos compostos por derivados orgânicos sulfurados (presentes como mercaptans, sulfetos, tiofenos, etc.), nitrogenados 
(presentes como piridina, pirrol, quinolina, porfirinas, etc.), oxigenados (presentes como ácidos carboxílicos e naftênicos, fenol, cresol) e organometálicos. Em geral, o petróleo é inflamável à temperatura ambiente, e suas propriedades físico-químicas apresentam grandes variações como densidades relativas entre 0,8 a 1,0 .

As propriedades físicas e termodinâmicas e o comportamento de uma mistura desse tipo dependem principalmente de seus constituintes, das quantidades relativas destes constituintes e das condições termodinâmicas nas quais ela se encontra.

Os hidrocarbonetos presentes no petróleo apresentam propriedades físicas bastante distintas entre si, o que se reflete sobre as propriedades físicas dos petróleos. Dependendo da proporção de hidrocarbonetos na sua composição, o petróleo se mostra mais adequado para a produção de um ou outro derivado. Assim, suas propriedades físicas podem variar muito de acordo com o tipo de hidrocarboneto predominante no petróleo podendo ser do tipo parafínico, naftênico ou aromático, segundo Szklo (2005).

Os componentes presentes no petróleo também podem ser agrupados em quatro classes principais, sendo este critério baseado na diferença de solubilidade, conhecido como análise SARA, que é um método de fracionamento no qual o petróleo é separado em saturados (alcanos e cicloparafinas), aromáticos (hirdrocarbonetos mono, di e poliaromáticos), resinas (frações constituintes de moléculas polares contendo heteroátomos $\mathrm{N}, \mathrm{O}$ ou $\mathrm{S}$, derivados de porfirina, além de apresentar traços de compostos como álcool, fenol, cetona e ácido carboxílico) e asfaltenos (são moléculas similares às resinas, porém possuindo maior massa molar e núcleo poliaromático, ricos também em metais).

Os diferentes tipos de petróleo são classificados, quanto à densidade, segundo uma gradação que vai de leves (menos densos) a pesados (mais densos). Essa classificação é convencionada de acordo com as normas do American Petroleum Institute, sendo por isso conhecida como "grau API". Quanto menor a densidade do petróleo maior o grau API e maior o seu valor comercial, pois neste tipo de petróleo é possível produzir, em princípio, uma parcela maior de derivados nobres, de elevado valor comercial, tais como gasolina, diesel e o GLP, relativamente a outro tipo de óleo mais pesado. O grau API é calculado a partir da Equação 1 extraída de Szklo (2005).

$$
{ }^{\circ} A P I=[141,5 / \text { gravidade especifica }]-131,5
$$

Onde a gravidade específica é a razão entre a densidade específica do material e a da água à mesma temperatura $\left(60^{\circ} \mathrm{F}\right)$.

\subsection{Os asfaltenos}

Existem dois grupos de partículas coloidais, os quais estão dissolvidos no meio de dispersão oleosa, sendo eles: os asfaltenos e as resinas, de acordo com Coelho (1992). Os asfaltenos, conforme mostra a Figura 1, são surfactantes naturais do petróleo formados predominantemente por anéis aromáticos policondensados e cadeia alifática laterais, ocorrendo em menor proporção, grupos funcionais ácidos e metais complexados. 


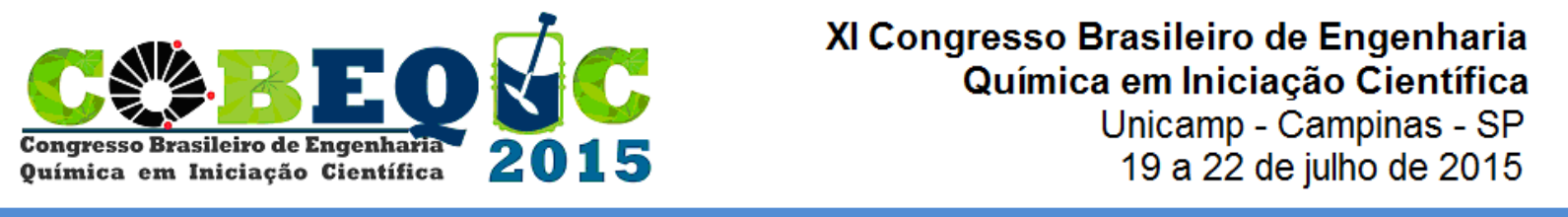

Figura 1 - Ilustração da estrutura molecular representativa para dois diferentes asfaltenos.

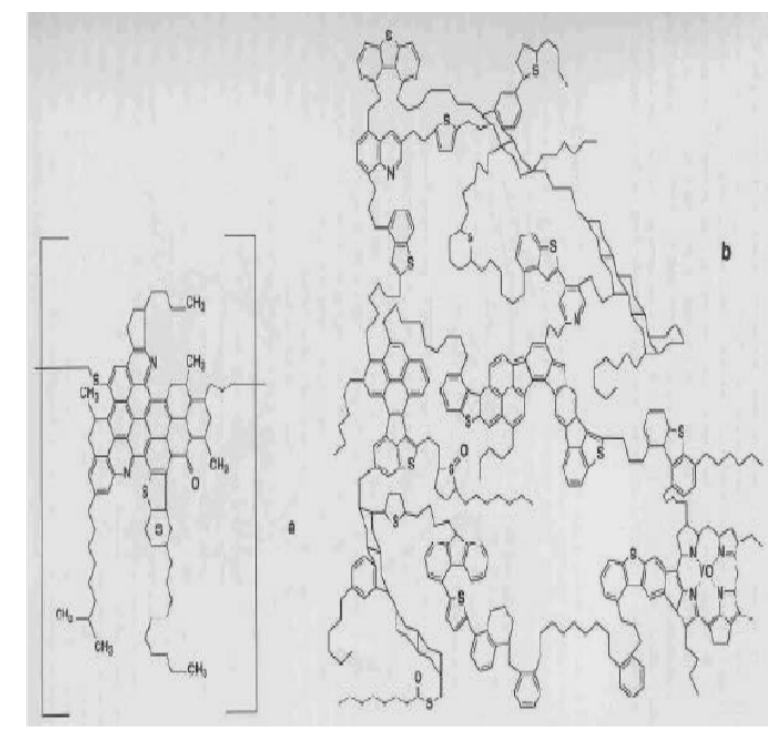

Os asfaltenos são definidos como compostos que são insolúveis em n-heptano e solúveis em tolueno. Existem dois modelos diferentes para a macroestrutura dos asfaltenos em solução. O modelo de Pfeiffer e Saal (1940) propõe que os asfaltenos são discos poliaromáticos contendo heteroátomos, intrinsecamente insolúveis no petróleo e em hidrocabonetos, porém mantidos em suspensão graças a solvatação de moléculas cada vez menos aromáticas e polares, num gradiente até moléculas com características próximas as do meio solvente. O outro modelo, proposto por Yen (1967), é bem mais complexo e envolve a participação de outras entidades moleculares, dentre as quais destacam-se as resinas. As resinas seriam substâncias não tão pesadas ou polares quanto os asfaltenos, nem teriam propensão a agregação, mas proporcionariam uma espécie de peptização dos asfaltenos, mantendo-os suspensos.

O problema da formação de materiais sólidos durante o processamento e estocagem do petróleo é causado principalmente por dois componentes do petróleo cru, as parafinas e os asfaltenos, segundo Manning e Thompson (1995), lembrando que cada um destes é uma mistura de componentes. Ambas as espécies podem precipitar como sólidos durante o tratamento do petróleo cru ou até mesmo no poço. A deposição destes sólidos é tão significante que até pode obstruir tubulações e equipamentos e, em casos extremos, cessar o fluxo e causar sérias dificuldades no escoamento.

\section{MATERIAIS E MÉTODOS}

Inicialmente foram realizados testes de solubilidade do óleo cru pesado, da Bacia de Campos do campo de Marlim, de grau API 19, densidade de $0,94 \mathrm{~g} / \mathrm{cm}^{3}$, a diferentes volumes do agente precipitante n-heptano (VETEC). Primeiramente, foram pesadas em balança analítica, 3 amostras com uma quantidade do óleo referente a aproximadamente $1 \mathrm{ml}$, e misturados a determinados volumes de n-heptano: $20 \mathrm{ml}, 40 \mathrm{ml}$ e $60 \mathrm{ml}$. Após 5 minutos em 
contato tem-se a formação de precipitado, sendo submetida à filtração a vácuo. $\mathrm{O}$ asfalteno precipitado, de coloração escura (preta), ficou retido no papel de filtro. Que foram colocados em estufa para secagem durante $12 \mathrm{~h}$, e já secos, pesados em uma balança analítica. O filtrado, livre de asfalteno, foi armazenado em um vial para posterior utilização.

Posteriormente verificou-se a influência de diferentes solventes na estabilidade coloidal do petróleo utilizando força centrífuga. Os solventes utilizados foram: n-heptano, tolueno, ciclohexano e xileno, todos grau PA (VETEC). Foram adicionados aos tubos de ensaio de massa igual a $10,75 \mathrm{~g}, 1 \mathrm{ml}$ de óleo cru pesado, equivalente a $0,94 \mathrm{~g}$, e a cada um $30 \mathrm{ml}$ de um determinado solvente (1:30) que foram centrifugados (centrífuga Hettich Zentrifugen D78532) a $2500 \mathrm{rpm}$, na temperatura ambiente. Para todos os solventes foram preparadas amostras em duplicata. Este procedimento foi realizado no tempo de 5 minutos. Após a centrifugação realizou-se filtração a vácuo em todas as amostras e os precipitados ficaram retidos nas paredes dos tubos, que foram pesados em balança analítica. Os filtrados foram armazenados em vials para posterior utilização.

O óleo desasfaltado pelo solvente n-heptano, anteriormente armazenado nos vials, foi submetido a análises cromatográficas, pela cromatografia gasosa, onde $1 \mu 1$ de amostra foi injetado no cromatógrafo gasoso HP 5890, utilizando uma microseringa de $10 \mu 1$. A coluna cromatográfica utilizada será a HP-5 (5\% Phenyl 95\% dimethylpolysiloxane) com dimensões de $30 \mathrm{~m} \times 0,32 \mathrm{~mm} \times 0,25 \mu \mathrm{m}$. A metodologia empregada foi baseada no método ASTM D5134, nas condições $35^{\circ} \mathrm{C}(30 \mathrm{~min}), 2^{\circ} \mathrm{C} / \mathrm{min}$ a $200^{\circ} \mathrm{C}$. A razão de split empregada foi de 1:100. Detector por ionização de chama (DIC).

\section{RESULTADOS E DISCUSSÕES}

No teste de solubilidade com n-heptano constatou-se que na proporção de 1:40 (petróleo/n-heptano) obteve-se maior porcentagem de asfalteno precipitado. Os resultados obtidos estão apresentados na Tabela 1.

Tabela 1 - Testes de solubilidade do óleo cru pesado em n-heptano, da Bacia de Campos do campo de Marlim, de grau API 19.

\begin{tabular}{|c|l|}
\hline Proporção $(\mathrm{ml})$ & \% de Asfalteno \\
\hline $1: 20$ & 0,68 \\
\hline $1: 40$ & 5,99 \\
\hline $1: 60$ & 3,97 \\
\hline
\end{tabular}

$\mathrm{Na}$ verificação da eficiência de solventes frente à separação de asfaltenos percebe-se que a floculação e, consequente, sedimentação do asfalteno ocorre devido ao rompimento do equilíbrio de solvatação provocado pela adição de um solvente apropriado. A estabilidade do precipitado formado depende tanto da natureza do solvente quanto da natureza do próprio petróleo, logo a separação do asfalteno variou de acordo com as propriedades do solvente uma 
vez que o petróleo utilizado foi o mesmo para todas as amostras. Foi possível verificar que o n-heptano é mais eficiente para precipitação de asfalteno, seguido do ciclohexano, xileno e por último tolueno, como é apresentado na Tabela 2. A porcentagem média de asfalteno obtido com o n-heptano utilizando força centrífuga foi de $46,50 \%$, bem superior ao obtido pelo método gravimétrico.

Tabela 2 - Verificação da eficiência de solventes frente à separação de asfaltenos.

\begin{tabular}{|c|c|}
\hline Solvente & $\%$ de Asfalteno \\
\hline n-heptano & $46,50 \pm 0,66$ \\
\hline Ciclohexano & $34,22 \pm 1,42$ \\
\hline Xileno & $30,24 \pm 0,33$ \\
\hline Tolueno & $23,85 \pm 0,80$ \\
\hline
\end{tabular}

$\mathrm{Na}$ análise cromatográfica do óleo desasfaltado pelo solvente n-heptano, utilizando o método ASTM D5134, os possíveis compostos a serem identificados estão na Figura 2.

Figura 2 - Cromatograma ASTM D5134.

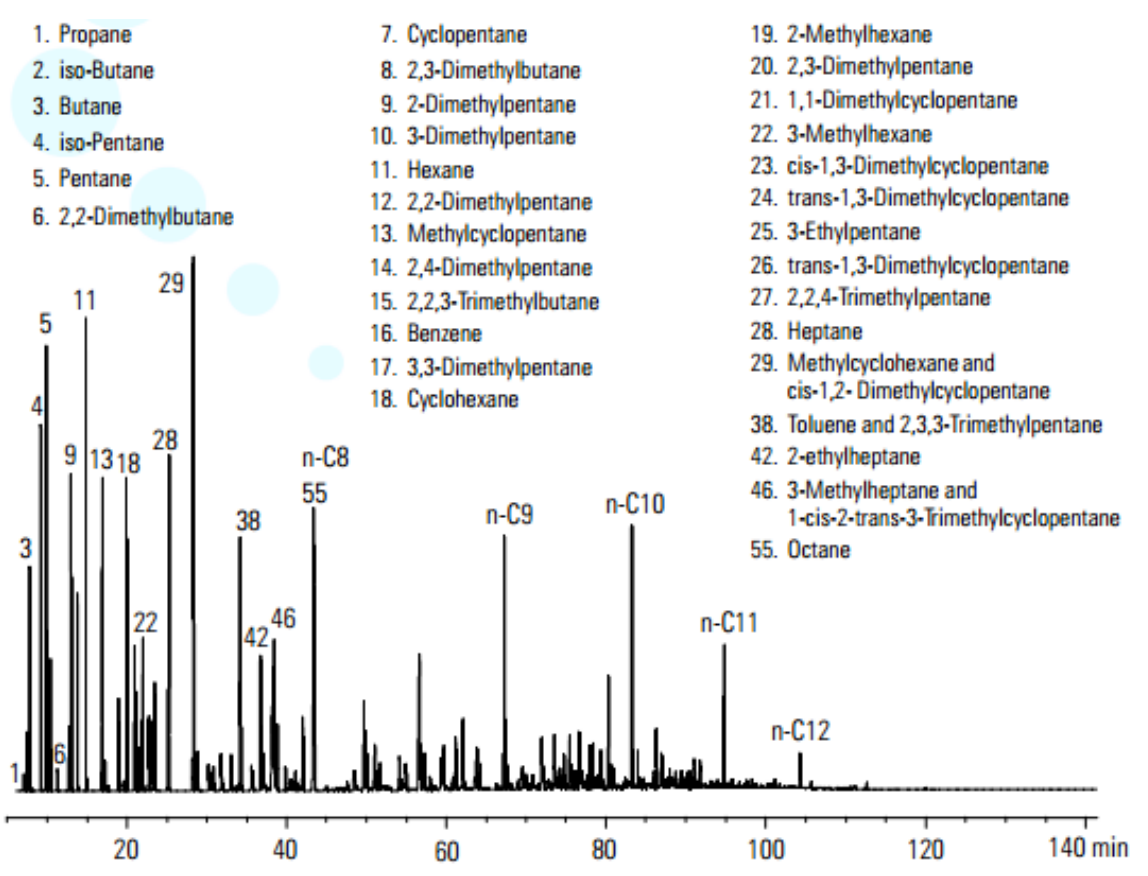

\section{CONCLUSÃO}

Com os resultados obtidos observa-se que os asfaltenos podem ser separados do óleo cru a partir de solventes orgânicos. Com a introdução de uma substância solubilizante é promovido um rompimento no equilíbrio do sistema, retirando as resinas que protegem os 
asfaltenos e permitindo a aproximação dos asfaltenos e a formação de partículas maiores até a sua precipitação. Foi possível constatar que a ordem de eficiência dos solvente frente à separação de asfalteno foi n-heptano>ciclohexano>xileno>tolueno. Ou seja, solventes de natureza parafínica são mais eficientes frente a separação do asfalteno. Este método mostra-se bastante eficaz na prevenção de formação de precipitados por asfaltenos na indústria de petróleo, reduzindo os problemas provocados por esta formação.

\section{REFERÊNCIAS}

COELHO, G. L. V. (1992), Desasfaltação com CO2. In: IX Congresso brasileiro de engenharia química, Salvador-Ba, Anais. Editora Abeq, 349-355;

MANNING, F.S.; THOMPSON, R.E. (1995), Oilfield processing, Volume two: Crude oil. Tulsa, OK; Pennwell Books, 2, 2, 13-15;

PFEIFFER, J. P., SAAL, N. R. J. (1940), J. Phys. Chem., 44, 139-149;

SZKLO, A.S.; (2005). Fundamentos do Refino do Petróleo. Rio de Janeiro: Interciência: PETROBRAS;

YEN, T. F., ERDMAN, J. G., POLLACK, S. S. (1967), Investigation of the structure of petroleum by X-ray diffraction. Anal Chem., 33, 11, 1587-1594.

Os autores agradecem a FAPERJ pelo apoio financeiro. 